\title{
GENERATION OF DIFFERENCE FREQUENCY RADIATION IN THE FIELD OF FEW-CYCLE LASER PULSE PROPAGATING IN GaAs CRYSTAL WITH PERIODIC AND APERIODIC DOMAIN STRUCTURE
}

\author{
D.L. HOVHANNISYAN ${ }^{*}$ \\ Radiophysics Department, Yerevan State University, \\ 1 A. Manoogian St., 375049 Yerevan, Armenia, Country, davidhl@ysu.am ${ }^{\dagger}$ \\ V.O. CHALTIKYAN \\ Institute for Physical Research, National Academy of Sciences, \\ Ashtarak, 378410 Armenia, chaltv@ipr.sci.am, \\ G.D. HOVHANNISYAN \\ Radiophysics Department, Yerevan State University, \\ 1 A. Manoogian St., 375049 Yerevan, Armenia, Country, gevorg199123@ysu.am
}

\begin{abstract}
We consider process of generation of difference frequency in a GaAs crystal with periodic domain structure during propagation of a few-cycle laser pulse in the crystal in the regime when chromatic dispersion is expressed weakly. Method of lines is used to obtain numerical solution to the system of coupled nonlinear differential equations in partial derivatives describing the evolution of the electric field of a few-cycle laser pulse in GaAs crystal both with periodic and aperiodic domain structure. Time-frequency distribution is obtained, with use of Wigner transformation, for linearlypolarized femtosecond laser pulse and for orthogonally polarized difference-frequency pulse generated via filtration of the orthogonally-polarized pulse at the crystal output.

Keywords: Femtosecond laser pulse; terahertz pulse; supercontinuum; Wigner transform; method of lines.
\end{abstract}

\section{Introduction}

In recent years considerable successes have been achieved in generation and detection of ultrashort terahertz pulses by optical methods, among which the most widespread became the techniques based on exploiting electro-optical materials ${ }^{1-3}$. In these materials generation of terahertz radiation may be considered as rectification of an optical pulse occurring at interaction of strong optical radiation with nonlinear medium. For efficient transformation of radiation of a femtosecond laser pulse into terahertz radiation the nonlinear-crystal-based periodic domain structures realizing the concept of phase quasisynchronism are widespread. Periodically polarized ferroelectric with regular onedimensional domain structure are the most promising ${ }^{4,5}$. For generation of difference frequency radiation an isotropic GaAs crystal with periodic domain structure is often 
used $^{6-8}$, which has a transparency band $0.917 \mu \mathrm{m}$ and the coefficient of absorption ${ }^{9}$ in the frequency range up to $3 \mathrm{THz}$ lower than $5 \mathrm{~cm}^{-1}$. The coefficient of nonlinear susceptibility of GaAs is sufficiently high and comparable with corresponding values for such crystals as $\mathrm{ZnTe}, \mathrm{GaP}$, and $\mathrm{GaSe}$, which also are used for difference frequency generation. We study in this paper the process of difference frequency generation by a femtosecond laser pulse propagating in GaAs crystal with both periodic and aperiodic domain structure. For description of this process in case of linearly polarized few-cycle laser pulse and in the regime of weakly expressed chromatic dispersion we use the system of coupled differential equations in partial derivatives valid in approximation of unidirectional waves. We obtain the numerical solution of this system by the method of lines. We calculate the temporal profile and the spectrum of the terahertz pulse produced via filtration of spectral supercontinuum formed in the output of nonlinear crystal. Time dependences of instantaneous frequencies of the terahertz pulse are obtained. We will show that employing aperiodically polarized domain structures allows controlling the law of frequency modulation of the broadband terahertz pulse. For study of time-frequency distribution of the broadband difference-frequency pulse the Wigner transformation (WT) will be used.

\section{Model of Polarization Response}

Let us consider the case where linearly polarized laser pulses with plane wavefronts and mutually orthogonal planes of polarization $E_{z}$ and $E_{y}$ are propagating along the $x$-axis normal to the $\langle 110\rangle$ plane in an isotropic GaAs crystal. The corresponding wave equations may be represented in the form

$\frac{\partial^{2} E_{z, y}}{\partial x^{2}}-\frac{1}{c^{2}} \frac{\partial^{2} E_{z, y}}{\partial t^{2}}=\frac{4 \pi}{c^{2}} \frac{\partial^{2} P_{L z,(L y)}}{\partial t^{2}}+\frac{4 \pi}{c^{2}} \frac{\partial^{2} P_{N L z,(N L y)}}{\partial t^{2}}$

where $\mathrm{P}_{\mathrm{Lz},(\mathrm{Ly})}$ and $\mathrm{P}_{\mathrm{NLz},(\mathrm{NLy})}$ are, respectively, linear and nonlinear parts of the medium polarization. The linear response of the medium to the $z-$ and y-polarizations is determined by the expressions

$$
P_{L z, L y}(\omega)=\varepsilon_{0} \chi^{(1)}(\omega) E_{z, y}(\omega)
$$

with $\varepsilon_{0}$ the vacuum dielectric constant and $\chi^{(1)}(\omega)$ the linear susceptibility of medium. The linear susceptibility of GaAs can in the spectral range of $0.19-17 \mu \mathrm{m}$ be represented $^{7}$ as

$\chi^{(1)}(\omega)=n^{2}(\omega)-1=b_{0}+\sum_{i=1}^{3} b_{i}(2 \pi c)^{2} /\left(\omega_{i}^{2}-\omega^{2}\right)$,

where $\mathrm{b}_{0}=4.372514, \mathrm{~b}_{1}=27.83972\left[\mu \mathrm{m}^{-2}\right], \mathrm{b}_{2}=0.031764\left[\mu \mathrm{m}^{-2}\right]+4.35 \cdot 10^{-5}\left[\mu \mathrm{m}^{-2} / \mathrm{K}\right] \cdot \Delta \mathrm{T}+$ $4.664 \cdot 10^{-7}\left[\mu \mathrm{m}^{-2} / \mathrm{K}^{2}\right] \cdot \Delta \mathrm{T}^{2}$,

$\mathrm{b}_{3}=0.00143636\left[\mu \mathrm{m}^{-2}\right], \lambda_{1}=\left(0.4431307[\mu \mathrm{m}]+0.50564 \cdot 10^{-4}[\mu \mathrm{m} / \mathrm{K}] \cdot \Delta \mathrm{T}\right), \quad \lambda_{2}=(0.8746453$ $\left.[\mu \mathrm{m}]+0.1913 \cdot 10^{-3}[\mu \mathrm{m} / \mathrm{K}] \cdot \Delta \mathrm{T}-4.882 \cdot 10^{-7}\left[\mu \mathrm{m} / \mathrm{K}^{2}\right] \cdot \Delta \mathrm{T}^{2}\right), \quad \lambda_{3}=(36.9166[\mu \mathrm{m}] \quad-$ $0.011622[\mu \mathrm{m} / \mathrm{K}] \cdot \Delta \mathrm{T}), \lambda_{\mathrm{i}}=2 \pi \mathrm{c} / \omega_{\mathrm{i}}, \mathrm{c}=3 \cdot 10^{14} \mu \mathrm{m} / \mathrm{sec}, \Delta \mathrm{T}$ is the temperature deviation from 
room temperature in Kelvin $-[\mathrm{K}]$, and $\mathrm{n}$ the medium refractive index. In the chosen geometry the nonlinear polarization of the medium caused by quadratic susceptibility may in the quasistatic approximation be written as

$$
P_{N L z}(t)=\varepsilon_{0} d_{14} E_{y}^{2}(t), \quad P_{N L y}(t)=\sqrt{2} \varepsilon_{0} d_{14} E_{z}(t) E_{y}(t),
$$

where $d_{14}=150 \cdot 10^{-12} \mathrm{~m} / \mathrm{V}$ is the coefficient of nonlinear susceptibility of GaAs crystal. Consider the case where spectra of both laser and difference frequency radiation lie below the electronic resonance frequencies, but above ionic resonance frequencies of the medium. Under these conditions the refractive index in (4) can be represented ${ }^{10}$ as a series

$\mathrm{n}(\omega, \Delta \mathrm{T})=\alpha(\Delta \mathrm{T})+\beta(\Delta \mathrm{T}) \mathrm{c} \omega^{2}+\gamma(\Delta \mathrm{T}) \mathrm{c} \omega^{4}-\theta(\Delta \mathrm{T}) \mathrm{c} / \omega^{2}$,

$$
\begin{aligned}
& \alpha(\Delta \mathrm{T})=\sqrt{\mathrm{a}_{0}(\Delta \mathrm{T})}, \beta(\Delta \mathrm{T})=\mathrm{a}_{1}(\Delta \mathrm{T}) / 2 \mathrm{c} \sqrt{\mathrm{a}_{0}(\Delta \mathrm{T}),} \\
& \gamma(\Delta \mathrm{T})=\mathrm{a}_{2}(\Delta \mathrm{T}) / 2 \mathrm{c} \sqrt{\mathrm{a}_{0}(\Delta \mathrm{T})}, \theta(\Delta \mathrm{T})=\mathrm{a}_{3} / 2 \mathrm{c} \sqrt{\mathrm{a}_{0}(\Delta \mathrm{T})},
\end{aligned}
$$

where

$$
\begin{aligned}
& \mathrm{a}_{0}(\Delta \mathrm{T})=1+\mathrm{b}_{0}+\mathrm{b}_{1}(2 \pi \mathrm{c})^{2} / \omega_{1}^{2}(\Delta \mathrm{T})+\mathrm{b}_{2}(\Delta \mathrm{T})(2 \pi \mathrm{c})^{2} / \omega_{2}^{2}(\Delta \mathrm{T}), \\
& \mathrm{a}_{1}(\Delta \mathrm{T})=\frac{\mathrm{b}_{1}(2 \pi \mathrm{c})^{2}}{\omega_{1}^{4}(\Delta \mathrm{T})}+\frac{\mathrm{b}_{2}(\Delta \mathrm{T})(2 \pi \mathrm{c})^{2}}{\omega_{2}^{4}(\Delta \mathrm{T})}, \mathrm{a}_{2}(\Delta \mathrm{T})=\frac{\mathrm{b}_{2}(\Delta \mathrm{T})(2 \pi \mathrm{c})^{2}}{\omega_{2}^{6}(\Delta \mathrm{T})}, \mathrm{a}_{3}=\mathrm{b}_{3}(2 \pi \mathrm{c})^{2} .
\end{aligned}
$$

In periodic domain structures the periodic change in sign of quadratic susceptibility occurs at interfaces between domains, which produce conditions for constructive interference of signal and idle waves in bulk crystals with arbitrary dispersion characteristics. The nonlinear susceptibility may in this case be written ${ }^{8}$ as

$$
\mathrm{d}_{14}(\mathrm{x})=\mathrm{d}_{14} \sum_{\mathrm{m}=0}^{\mathrm{M}} \frac{\sin (2 \pi \mathrm{x}[2 \mathrm{~m}+1] / \Lambda)}{(2 \mathrm{~m}+1)} \frac{\sin [\pi(\mathrm{m}+1) / \mathrm{M}]}{\pi(\mathrm{m}+1) / \mathrm{M}} \text {, }
$$

where $\Lambda$ is the period of the domain structure, $m=0,1,2, \ldots, M$, and $M$ is the number of terms in the sum (8). Obviously, the nonlinear part of medium polarization will also be a periodic function of the $\mathrm{x}$-coordinate. The value of the period should be determined by the energy and momentum conservation laws

$$
1 / \lambda_{p}=1 / \lambda_{s}+1 / \lambda_{T H z}, n\left(\lambda_{p}\right) / \lambda_{p}=n\left(\lambda_{s}\right) / \lambda_{s}+n\left(\lambda_{T H z}\right) / \lambda_{T H z}+1 / \Lambda,
$$

with $\lambda_{p}$ and $\lambda_{s}$ being, respectively, the short and the long wavelengths, within the spectral band of the few-cycle laser pulse, whose interaction in quadratically nonlinear medium may lead to difference frequency $\left(\lambda_{\mathrm{THz}}\right)$ generation. In the periodic domain structures the quasi-phase matching is realized for all pairs of $\lambda_{\mathrm{p}}$ and $\lambda_{\mathrm{s}}$ spectral components satisfying the condition (9). In addition to spectral components within the width of the initial pulse, one should take into account also the new components produced in nonlinear interaction of the initial pulse with the medium. In particular, for a laser pulse with Gaussian time profile, duration $\tau_{0}=30 \mathrm{fs}$, central wavelength $\lambda_{0}=1.98 \mu \mathrm{m}$, and the spectrum width 
$\Delta v=(2 \ln 2) 1 / 2 /\left(\pi \tau_{0}\right)=24.99 \mathrm{THz}(\Delta \lambda=329 \mathrm{~nm})$, spectral components from $1.813 \mu \mathrm{m}$ to $2.144 \mu \mathrm{m}$ are within the spectral bandwidth. It is known that the use of aperiodic domain structures leads to broadening of the quasi-phase matching band along with some decrease in the efficiency of the difference frequency generation ${ }^{4,5}$. We consider below aperiodic domain structures which allow producing broadband difference frequency radiation. In particular, structures with both positive and negative spatial chirp of the period will be considered. Domain thickness of these chirped aperiodic media may be represented as

$$
\Delta_{\mathrm{i}}=\Delta_{\max }-\mathrm{i} \delta \Delta, \quad \text { (10) } \Delta_{\mathrm{i}}=\Delta_{\min }+\mathrm{i} \delta \Delta
$$

where $\Delta_{\max }$ and $\Delta_{\min }$ are the maximum and minimum values of the structure thickness, $\mathrm{i}=0,1, \ldots, 2 \mathrm{~N}$ ( $\mathrm{N}$ the number of domains), and $\delta \Delta=\left(\Delta_{\max }-\Delta_{\min }\right) /(\mathrm{N}-1)$. Conditions (10) and (11) correspond to media with, respectively, negative and positive spatial chirp. Equations (1) and (2) in the approximation of unidirectional waves were $\operatorname{shown}^{10}$ to be representable, in normalized form and with use of (6), as

$$
\begin{aligned}
& \frac{\partial \Phi_{\mathrm{z}}}{\partial \xi}-\frac{\partial^{3} \Phi_{\mathrm{z}}}{\partial \eta^{3}}+\mathrm{A} \frac{\partial^{5} \Phi_{\mathrm{z}}}{\partial \eta^{5}}+\mathrm{B} \int_{-\infty}^{\eta} \Phi_{\mathrm{z}} \mathrm{d} \eta+4 \pi \mathrm{C}(\xi) \Phi_{\mathrm{y}} \frac{\partial \Phi_{\mathrm{y}}}{\partial \eta}=0, \\
& \frac{\partial \Phi_{\mathrm{y}}}{\partial \xi}-\frac{\partial^{3} \Phi_{\mathrm{y}}}{\partial \eta^{3}}+\mathrm{A} \frac{\partial^{5} \Phi_{\mathrm{y}}}{\partial \eta^{5}}+\mathrm{B} \int_{-\infty}^{\eta} \Phi_{\mathrm{y}} \mathrm{d} \eta+2 \sqrt{2} \pi \mathrm{C}(\xi) \Phi_{\mathrm{z}} \frac{\partial \Phi_{\mathrm{y}}}{\partial \eta}+ \\
& 2 \sqrt{2} \pi \mathrm{C}(\xi) \Phi_{\mathrm{y}} \frac{\partial \Phi_{\mathrm{z}}}{\partial \eta}=0,
\end{aligned}
$$

where the time and space variables are defined as $\tau=t-\alpha(\Delta T) x / c, \eta=2 \pi \tau / T_{0}=\omega_{0} \tau=2 \pi \lambda_{0} \tau / \mathrm{c}$, $\xi=x \beta^{\prime}(\Delta \mathrm{T})=x \omega_{0}{ }^{3} \beta(\Delta \mathrm{T})$, the field components are normalized to the maximum value of the electric field amplitude, $\Phi_{\mathrm{z}, \mathrm{y}}=\mathrm{E}_{\mathrm{z}, \mathrm{y}} / \mathrm{E}_{0 \max }$, and the coefficients $\mathrm{A}, \mathrm{B}$, and $\mathrm{C}(\xi)$ are defined by the formulas $A=\omega_{0}{ }^{2} \gamma / \beta=\omega_{0}{ }^{2} a_{2} / a_{1}, \quad B=\left(1 / \omega_{0}{ }^{4}\right) \theta / \beta=\quad\left(1 / \omega_{0}{ }^{4}\right) a_{3} / a_{1}$, $\mathrm{C}(\xi)=\left(1 / c \omega_{0}^{2}\right) \mathrm{d}_{14}(\xi) \mathrm{E}_{0 \max } / \alpha \beta=\left(2 / \omega_{0}^{2}\right) \mathrm{d}_{14}(\xi) \mathrm{E}_{0 \max } / \mathrm{a}_{1}$. Coefficient $\mathrm{A}$ in Eqs. (12), (13) equals ${ }^{10}$ the ratio of the length of second order dispersion spreading $L_{d 2}=2 c\left(a_{0}\right)^{1 / 2} /\left(\omega_{0}{ }^{3} a_{1}\right)$ to that of fourth order dispersion spreading $L_{d 4}=2 c\left(a_{0}\right)^{1 / 2} /\left(\omega_{0}{ }^{5} a_{2}\right)$. Factor $B$ is the ratio of $\mathrm{L}_{\mathrm{d} 2}$ to the length of dispersion spreading caused by the ionic linear polarization response $\mathrm{L}_{\mathrm{i}}=2 \omega_{0} \mathrm{c}\left(\mathrm{a}_{0}\right)^{1 / 2} / \mathrm{a}_{1}$. The quantity $\mathrm{C}$ is the ratio of $\mathrm{L}_{\mathrm{d} 2}$ to the characteristic length of nonlinear interaction $\mathrm{L}_{\mathrm{n}}=\mathrm{c}\left(\mathrm{a}_{0}\right)^{1 / 2} /\left(2 \omega_{0} \mathrm{~d}_{14} \mathrm{E}_{0 \max }\right)$. In case of GaAs crystal, for $\lambda_{0}=1.98 \mu \mathrm{m}$ and temperature $22^{\circ} \mathrm{C}$, we obtain $\mathrm{L}_{\mathrm{d} 2}=7.457 \mu \mathrm{m}, \mathrm{L}_{\mathrm{d} 4}=2.245 \mathrm{~mm}, \mathrm{~L}_{\mathrm{i}}=368.899 \mu \mathrm{m}$, and $\mathrm{L}_{\mathrm{n}}=34.62 \mu \mathrm{m}$ for $\mathrm{E}_{0 \max }=100 \mathrm{MV} / \mathrm{m}(\mathrm{A}=0.003322, \mathrm{~B}=0.02$, and $\mathrm{C}=4.643)$. For numerical integration of the system of equations (12) and (13) we choose the boundary conditions

$\Phi_{\mathrm{y}}(\xi=0, \eta)=\Phi_{\mathrm{y} 0} \exp \left(-\eta^{2} / \tau_{\mathrm{p}}^{2}\right) \cos (\eta), \Phi_{\mathrm{z}}(\xi=0, \eta)=0$

where $\tau_{\mathrm{p}}=15 \mathrm{fs}\left(\lambda_{0}=1.98 \mu \mathrm{m}\right)$ and $\Phi_{\mathrm{y} 0}$ is the normalized initial value of the amplitude of $y$-polarized pulse. During propagation of y-polarized laser pulse in the crystal a zpolarized pulse is produced ${ }^{10}$ whose spectrum contains components at sum and difference 
frequencies which is caused by the last term in (12). For numerical simulation we choose the period of the regular domain structure $\Lambda=10 \mathrm{~L}_{\mathrm{d} 2}=74.57 \mu \mathrm{m}$ and the length of nonlinear crystal $10 \Lambda=745.7 \mu \mathrm{m}$. The highest value of the input pulse amplitude is taken to be $\mathrm{E}_{0 \max }=100 \mathrm{MV} / \mathrm{m}$. According to (9), the blue component $\lambda_{\mathrm{p}}=1.894 \mu \mathrm{m}$ for the case $\lambda_{\mathrm{s}}=\lambda_{\mathrm{p}}+4 \Delta \lambda / 3$ and $\lambda_{\mathrm{p}}=1.815 \mu \mathrm{m}$ for the case $\lambda_{\mathrm{s}}=\lambda_{\mathrm{p}}+\Delta \lambda$ correspond to the chosen value of $\Lambda$ and according to (9) this gives the wavelengths of difference frequency radiation $\lambda_{\mathrm{THz}}=9.8 \mu \mathrm{m}$ for the first case and $\lambda_{\mathrm{THz}}=11.82 \mu \mathrm{m}$ for second case. For modeling the process of generation of difference frequency radiation we consider aperiodic domain structure (10) with negative spatial chirp with the domain thickness varying from $\Delta_{\max }=44.74 \mu \mathrm{m}$ to $\Delta_{\min }=29.83 \mu \mathrm{m}$. The number of domains is taken to be 20 , so that the domain thickness decreases from a domain to the next one by $1.65 \mu \mathrm{m}$. We also consider the domain structure with positive chirp (11) when the thicknesses of 20 domains increase from $29.83 \mu \mathrm{m}$ to $44.74 \mu \mathrm{m}$ by $1.65 \mu \mathrm{m}$. The choice of the domain structure, the nonlinear crystal, and the maximum value of the laser field amplitude is determined by the conditions of validity of the method of unidirectional waves, i.e., weak chromatic dispersion and small nonlinearity. The scheme of numerical solution of equations (12) and (13) by the method of lines ${ }^{11}$ is described in detail earlier ${ }^{10}$. The relative error in computation was chosen to be $10^{-6}$. In order to study the spectrum dynamics we use the Wigner transformation which has sufficient resolution in the time-frequency plane and allows revealing effectively ${ }^{12}$ the peculiarities of the time-frequency structure of the pulse.

\section{Computation Results and Discussion}

Here we describe the results of time-frequency analysis obtained with use of WT for zpolarized pulse traveling in GaAs crystal with periodic domain structure and which formed via filtration. For separation of difference frequency radiation from pumping radiation at $1.98 \mu \mathrm{m}$, it is necessary to transmit the $\mathrm{z}$-polarized radiation from output of nonlinear crystal through a silicon or germanium filter $1^{3}$. In numerical simulations the $\mathrm{z}-$ polarized pulse is filtered at the crystal output by a low-frequency filter with the coefficient of transmission $1 /\left(1+\left(v / v_{\mathrm{s} 0}\right)^{6}\right)$ where $v_{\mathrm{s} 0}=108.18 \mathrm{THz}\left(\lambda_{\mathrm{s} 0}=2.77 \mu \mathrm{m}\right)$. So, the filter transmits only the difference-frequency radiation. Figure 1 demonstrates the temporal profile, the spectral density, and the Wigner function of the z-polarized pulse at the output of nonlinear crystal with periodic domain structure having ten periods. The Wigner function is defined as

$$
\mathrm{W}_{\xi}(\eta, \tilde{\omega})=\int_{-\infty}^{\infty} \Phi_{\mathrm{y}}\left(\xi, \eta+\eta_{1} / 2\right) \Phi_{\mathrm{y}}^{*}\left(\xi, \eta-\eta_{1} / 2\right) \exp \left(-\mathrm{j} \tilde{\omega} \eta_{1}\right) \mathrm{d} \eta_{1},
$$

where $\tilde{\omega}=\omega / \omega_{\mathrm{s}}$ is the normalized frequency. Sampling frequency $\mathrm{F}_{\mathrm{s}}=2 \pi \omega_{\mathrm{s}}$ corresponding to the results shown in Fig. 1 equals $2.0084 \cdot 10^{15} \mathrm{~Hz}$. It is seen that the spectral maximum of difference frequency radiation lies at $31.131 \mathrm{THz}(9.6367 \mu \mathrm{m})$ and the instantaneous 
frequency increases nonlinearly from $20.084 \mathrm{THz}(14.937 \mu \mathrm{m})$ to $40.168 \mathrm{THz}$ $(7.4686 \mu \mathrm{m})$.

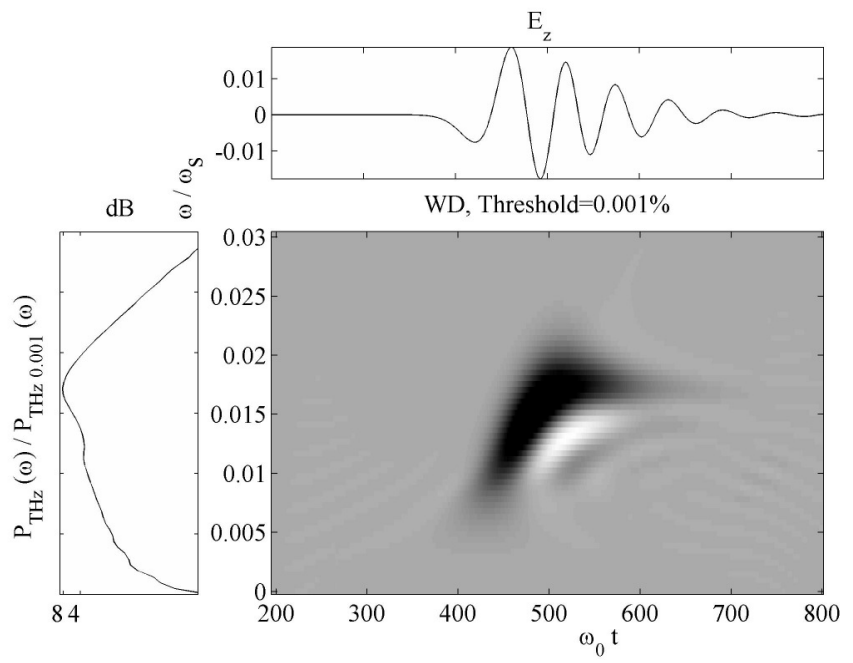

Fig. 1 Temporal profile, spectral density. and WD function of z-polarized difference-frequency pulse at the output of nonlinear crystal with 10-period domain structure.

This means that the difference frequency pulse has a nonlinear positive chirp and its spectral density goes from 0 to $56.236 \mathrm{THz}(5.3347 \mu \mathrm{m})$ at the level of 0.001 of the peak of Wigner distribution. Figure 2 demonstrates the temporal profile, spectral density, and Wigner distribution function for z-polarized filtered difference frequency pulse at the output of a crystal with aperiodic domain structure consisting of 20 domains with linearly decreasing thickness (10). The domain thickness decreases from 44.74 to $29.83 \mu \mathrm{m}$ with the step of $1.65 \mu \mathrm{m}$. It is seen that the peak in the difference frequency radiation spectrum lies at $40.331 \mathrm{THz}(7.4386 \mu \mathrm{m})$ and the instantaneous frequency increases in 100 fs from $20.165 \mathrm{THz}(14.877 \mu \mathrm{m})$ to $40.331 \mathrm{THz}(7.4386 \mu \mathrm{m})$, then decreases to $20 \mathrm{THz}(15 \mu \mathrm{m})$. So, the pulse of difference frequency radiation exhibits quadratic law of frequency modulation and the spectral density at the level 0.001 of the peak of Wigner distribution goes from 2 to $72.3 \mathrm{THz}(4.1493 \mu \mathrm{m})$. In this case, as distinct from the case of regular periodic domain structure, every domain has according to (9) its own value of the maximal instantaneous difference frequency. So, the domain with $\Delta_{\max }=44.74 \mu \mathrm{m}$ generates at $14 \mu \mathrm{m}$ for $\lambda_{\mathrm{s}}=\lambda_{\mathrm{p}}+\Delta \lambda$, while the domain with $\Delta_{\min }=29.83 \mu \mathrm{m}$ generates at $11.06 \mu \mathrm{m}$ for $\lambda_{\mathrm{s}}=\lambda_{\mathrm{p}}+\Delta \lambda$ and at $8.76 \mu \mathrm{m}$ for $\lambda_{\mathrm{s}}=\lambda_{\mathrm{p}}+4 \Delta \lambda / 3$. As seen in Fig. 2 , short-wave components in the time profile of the difference frequency pulse pass ahead of long-wave components. The efficiency of difference frequency generation with use of aperiodic domain structure with negative chirp (10) is $0.032 \%$. The same quantities, as in Fig.2, we plotted in Fig.3, but for aperiodic 20 domain structure with linearly increasing domain thickness (11). The thickness increases from $\Delta_{\min }=29.83 \mu \mathrm{m}$ to $\Delta_{\max }=44.74 \mu \mathrm{m}$ with the step of $1.65 \mu \mathrm{m}$. The peak is observed at $38.08 \mathrm{THz}(7.8782 \mu \mathrm{m})$ and the instantaneous 
frequency increases nonlinearly from $20 \mathrm{THz}(15 \mu \mathrm{m})$ to $42.176 \mathrm{THz}(7.113 \mu \mathrm{m})$. This means that the instantaneous difference frequency is a monotonic function of time and the spectral density at the level 0.001 of the peak of Wigner distribution goes from 2 to $58.244 \mathrm{THz}(5.15 \mu \mathrm{m})$.

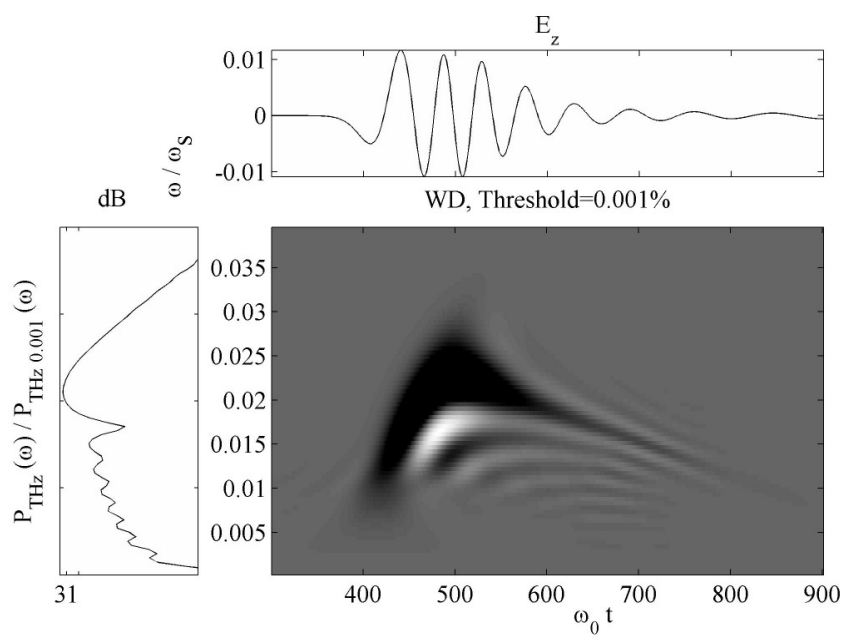

Fig. 2 Temporal profile, spectral density. and WD function of z-polarized filtered difference-frequency pulse at the output of crystal with aperiodic domain structure consisting of 20 periods with negative spatial chirp.

In this case long-wave components pass ahead of short-wave components. The efficiency of generation is in case (11) $0.067 \%$. We thus observe that if we use crystals with aperiodic domain structure, the efficiency of difference frequency generation is lower than in case of regular periodic structure (by a factor of 0.32 in case of negative spatial chirp and 0.67 in case of positive).

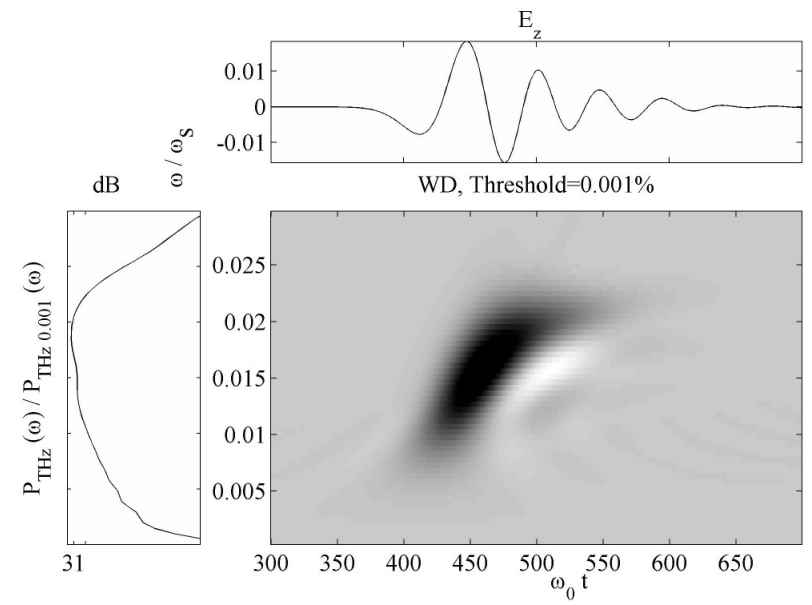

Fig.3 Temporal profile, spectral density and WD function of z-polarized filtered difference-frequency pulse at the output of crystal with aperiodic domain structure consisting of 20 periods with positive spatial chirp. 


\section{Conclusion}

We considered the generation of difference frequency radiation in a GaAs crystal with periodic and aperiodic domain structure in the field of a few-cycle laser pulse in the regime of weak chromatic dispersion. By means of Wigner transformation we have studied the time-frequency distribution for both y-polarized laser pulse and z-polarized difference frequency pulse. It was shown that the efficiency of different frequency generation is $0.1 \%$ for a 30 fs pulse at $1.98 \mu \mathrm{m}$ with the electric field amplitude of 100 $\mathrm{MV} / \mathrm{m}$ in GaAs crystal with ten periods of domain structure. We also have shown that this efficiency amounts to $0.032 \%$ in a negative-chirp aperiodic crystal and $0.064 \%$ with a positive-chirp crystal. The obtained results may be used for elaboration of a source of broadband terahertz radiation.

\section{References}

1. Y. Lee, Principles of Terahertz Science and Technology, (Springer: Berlin, 2009).

2. K. Sakai, Terahertz Optoelectronics, (Springer: Berlin, Heidelberg, 2005).

3. Q. Wu, X.C. Zhang, IEEE Journal of Selected Topics in Quantum Electronics, 2, 693 (1996).

4. J. A. L'huillier, G. Torosyan, M. Theuer, Yu. Avetisyan, R. Beigang, Appl. Phys. B, 86, 185 (2007).

5. J.A. L'huillier, G. Torosyan, M. Theuer, C. Rau, Yu. Avetisyan, R. Beigang, Appl. Phys. B, 86, 197 (2007).

6. Yun-Shik Lee, W.C. Hurlbut, K. L. Vodopyanov, M. M. Fejer, V. G. Kozlov, Applied Physics Letters, 89, 181104 (2006).

7. K.L. Vodopyanov, Optics Express, 14, 6, 2263 (2006).

8. D. L. Hovhannisyan, A.A. Hakhoumian, R.M. Martirosyan, A.S. Nikoghosyan, E.M. Laziev, G.D. Hovhannisyan, Journal of Modern Optics, 57, 12, 10, 1075 (2010).

9. T. Skauli, P.S. Kuo, K.L. Vodopyanov, T.J. Pinguet, O. Levi, L.A. Eyres, J. S. Harris, M. M. Fejer, E.L. Ginzton, B. Gerard, L. Becouarn, E. Lallier, Appl. Phys., 94, 10, 6447 (2003).

10. D. L. Hovhannisyan, V.O. Chaltykyan, A.S. Martirosyan, G.D. Hovhannisyan, K. A. Hovhannisyan, Journal of Contemporary Physics (Armenian Academy of Sciences), 46, 2, 58 (2011).

11. W.E. Schiesser, G.W. Griffiths, A Compendium of Partial Differential Equation Models, Method of Lines Analysis with Matlab, (Cambridge University Press: New York, 2009).

12. L. Cohen, Time-Frequency Analysis, (Prentice Hall PTR, Upper Saddle River, New Jersey, 1995).

13. T. Zentgraf, R. Huber, N.C. Nielsen, D.S. Chemla, R.A. Kaindl, Optics Express, 15, 9, 5775 (2007). 